\section{Alternativas para el mejoramiento urbano incluyente del asentamiento humano Alcázar del Café}

\section{Inclusive urban improvement alternatives for the human settlement Alcázar del Café}

\author{
Yonier Castañeda Pérez*, Naren Sebastián Salazar Londoño**
}

\begin{abstract}
*Arquitecto, Especialista en Gestión de Proyectos de Desarrollo y en Pedagogía y Docencia Universitaria, Magíster en Medio Ambiente y Desarrollo Sostenible. Hace parte de la Facultad de Arquitectura de la Universidad La Gran Colombia seccional Armenia como Docente Investigador. Miembro del Grupo de Investigación en Gestión Integral del Territorio. castanedapyonier@miugca.edu.co Armenia, Quindío, Colombia. Grupo: Territorio y arquitectura sustentable. ** Estudiante de Arquitectura, Universidad La Gran Colombia, semillero de investigación Mejoramiento urbano incluyente en asentamientos humanos
\end{abstract}

\section{Resumen}

La finalidad de la investigación fue llegar por medio del análisis de múltiples variables del territorio a propuestas y estrategias de mejoramiento urbano arquitectónico para un asentamiento humano, tomando como base las problemáticas que este presenta, formulando alternativas colectivamente acordadas desde una mirada arquitectónica que permita el mejoramiento de las condiciones de habitabilidad, los espacios colectivos y la calidad de vida de sus habitantes; a su vez, contribuir con la comunidad en el proceso de legalización del barrio.

Para abordar el tema se utilizó una metodología dividida en cuatro fases; la primera se enfocó en la aproximación al barrio y su reconocimiento empírico; en la segunda se realizó un diagnóstico completo del territorio; en la tercera fase se interactuó con las personas del asentamiento para formular propuestas y luego direccionar su aplicabilidad en la fase final.

Durante la investigación se identificó principalmente que los habitantes de esta urbanización tienen un deficiente uso y apropiación de los espacios públicos existentes, lo que genera que la comunidad no tenga buenas relaciones entre sí; además es notoria la desarticulación de los entes gubernamentales con las necesidades reales de la comunidad lo que finalmente motiva la participación de la academia en la búsqueda de concretar procesos entre los diferentes actores que inciden en este territorio.

Palabras clave: Asentamientos humanos, calidad de vida, mejoramiento integral de barrios, participación social, territorio

\begin{abstract}
The aim of this research was to reach from the analysis of multiple variables of the territory to the proposals and strategies for the urban-architectural improvement for the human settlement, based on its present problems, through the formulating of collectively agreed alternatives from an architectural perspective that allows the improvement of living conditions, collective spaces and quality of life of its inhabitants; at the same time, contribute to the community in the process of legalization of the neighborhood.

To address the process a methodology divided into four phases or moments was used; the first was focused on the approach to the neighborhood and its empirical recognition; in the second phase a complete diagnosis of the territory was carried out; in the third phase there was in interaction with the neighbors to make proposals and then address their applicability in the final phase.

During the research was identified mainly that the inhabitants of this urbanization have a poor use and appropriation of existing public spaces, what makes the community does not have good relations each other; besides is evident the disarticulation between the government agencies with the real needs of the community what ultimately encourages participation of academia in the pursuit of specify processes among the different actors involved in this territory.
\end{abstract}

Keywords: Human settlements, quality of life, human settlement upgrading, social engagement, territory
Recibido: $12 / 02 / 2015$

Revisado: 26/03/2015

Aceptado: 21/11/2015

Correspondencia de autor:

castanedapyonier@miugca.edu.co

(C) 2015 Universidad La Gran Colombia. Este es un artículo de acceso abierto, distribuido bajo los términos de la licencia Creative Commons Attribution License, que permite el uso ilimitado, distribución y reproducción en cualquier medio, siempre que el autor original y la fuente se acrediten.

* Artículo resultado del proyecto de investigación: Alternativas de mejoramiento urbano incluyente en asentamientos humanos informales o formales con desarrollo inadecuado de Armenia, Quindío - Colombia.

\section{Cómo citar:}

Castañeda, Y., Salazar, N.S. (2015) Alternativas para el mejoramiento urbno incluyente del asentamiento humano Alcázar del Café. UGCiencia 21, 60-72. 


\section{Introducción}

Se presenta una investigación que buscó la identificación, análisis e intervención de una realidad, con el fin de brindar una visión académica de las condiciones sociales y de habitabilidad que se viven o evidencian por parte de los habitantes de asentamientos humanos informales o formales con desarrollo inadecuado. Esta comprensión de la realidad vivida por algunas comunidades permitió llegar a lineamientos arquitectónicos y urbanísticos que toman en cuenta la calidad de vida, las necesidades y las formas de satisfacerlas, así como factores ambientales y de gestión comunitaria, todo ello para propender por un mejoramiento en las condiciones de vida más básicas en este tipo de entornos urbanos.

La temática de los barrios con problemas de legalización, falta de infraestructura y deficiencias en la apropiación del espacio público para las comunidades que los habitan, es una situación de la cual no está exenta ninguna ciudad del país. La presente investigación se propuso llevar un proceso que diera paso, tanto a la formulación de alternativas para el mejoramiento urbano como a la legalización de la urbanización Alcázar del Café de la ciudad de Armenia, necesidades relevantes para sus habitantes desde el mismo momento de su construcción.

Esta investigación participativa fue llevada a cabo por medio de un análisis físico-espacial, social, ambiental, normativo y de gestión donde se logró evidenciar las principales necesidades en cuanto a alternativas de mejoramiento barrial del sector de estudio, esto conllevó a un resultado eficaz y contextualizado que propone alternativas urbano arquitectónicas para mejorar algunas de las actuales condiciones de habitabilidad, a la vez que fortaleció la relación entre la academia, el gobierno local y la sociedad con la ejecución de acciones conjuntas que responden al entorno a intervenir; vinculando los distintos intereses, recursos y nexos de cada actor, para obrar de manera articulada en el aprovechamiento de oportunidades y posibilidades, la integración de iniciativas y capacidades de la comunidad.

El proceso se abordó con cuatro objetivos específicos traducidos en sus respectivas fases de desarrollo; comenzando por la aproximación a la comunidad donde se documentaron múltiples variables de indagación, esto con el fin de identificar la realidad urbana presente y analizar las condiciones de habitabilidad; posteriormente, en una segunda fase, se analizaron dichas variables para llegar a diagnosticar las problemáticas y oportunidades existentes que direccionan un tercer momento, el de formulación, allí se exploraron de forma participativa alternativas y estrategias para dar una respuesta urbano-arquitectónica a la comunidad ante sus necesidades sociales, físico-espaciales y de seguridad. El proceso finalizó con la fase de aplicación donde al tener una respuesta a las problemáticas y necesidades, se direcciona la gestión necesaria para la materialización de las propuestas.

De igual manera, este proceso produjo y aportó desde la mirada de futuros profesionales de la arquitectura un impacto positivo en las comunidades, dada la inclusión de la misma en todo el proceso investigativo, de manera objetiva y consiente a su realidad particular, convirtiéndola en el sujeto no solo beneficiario de los resultados, sino también como una parte activa dentro del mismo.

Finalmente, cabe mencionar que con esta investigaciónparticipativa se buscó contribuir a la comunidad del asentamiento humano en el proceso de legalización del mismo ya que por la falta de conocimientos urbanísticos y arquitectónicos y de información que viabilice la documentación y gestión de este tipo de procesos, se ven limitados para adelantarlo de una manera autónoma, esto por medio de la estructuración de las herramientas necesarias que le permita a la comunidad acceder a los beneficios sobre los cuales tienen derecho. Todo lo anterior hace que esta investigación se convirtiera en una herramienta de apoyo estratégico y funcional a programas institucionales del gobierno local, sirviendo a su vez como fundamento replicable de actuación y documentación frente planteamientos proyectuales similares.

\section{Desarrollo}

Esta investigación se inscribe principalmente como una Investigación de Campo de tipo mixto, dado que fue aplicada para interpretar y solucionar una situación, problema o necesidad en el marco del mejoramiento urbano y barrial y se desarrolló en un ambiente social en el que estuvieron presentes las personas, grupos y organizaciones comunitarias las cuales se convirtieron en la principal fuente de datos para ser analizados; así mismo, fue desarrollada con metodología de la Investigación Acción - Participación (IAP) cuyo planteamiento central es la acción trasformadora de una realidad identificada, con base en un análisis crítico con la participación activa 
de los grupos implicados, "que se orienta a estimular la práctica trasformadora y el cambio social proporcionando lineamientos para un mejor análisis y comprensión de la realidad que se hace evidente a través de problemas, oportunidades y necesidades" (Bru Martín y Basagoiti ,2003). El análisis de la información se realizó a parir de 4 componentes principales (Físico-espacial, Socio-económico, Ambiental y Normativo) y 14 variables de indagación lo que arrojó resultados tanto planimétricos como estadísticos. Se aplicaron también actividades de cartografía social a grupos focales.

Con el fin de tener una clara percepción del asentamiento se llevó a cabo la recopilación de datos a partir de fichas de registro, encuestas y entrevistas aplicadas, bien sea en el sector mismo, o con los habitantes de la urbanización. Como referente principal en la estructuración de varios de los instrumentos aplicados se tomó como base el proceso investigativo de la línea de investigación en Mejoramiento Integral Barrial (MIB) de la Universidad Pontificia Bolivariana de Medellín (UPB) junto con el Área Metropolitana del Valle de Aburrá (2007), quienes han adelantado desde el año 2004 propuestas hacia estrategias sobre vivienda y hábitat, basando su desarrollo en cuatro fases de diseño: recolectar e inventariar información, identificar problemáticas y oportunidades, definir estrategias de intervención y por ultimo diseñar y detallar el proyecto identificado en la etapa anterior. Por su parte, como referente principal para la aplicación de herramientas para el diagnóstico y diseño participativo se extrajo de la metodología denominada Change by Desing (Apsan Frediani, French, \& Nuñez Ferrera, 2011) de la Organización No Gubernamental Arquitectos Sin Fronteras del Reino Unido (ASK-UK), metodología que ha sido elaborada para el mejoramiento de barrios informales y el fomento del derecho a viviendas dignas, tomando en cuenta las complejidades sociales, económicas y políticas características del entorno en diferentes ciudades del mundo.

La comunidad en su totalidad y el mismo sector de la urbanización Alcázar del Café localizada al sur de la ciudad de Armenia, Quindío (Imagen 1) en la comuna 2, Rufino José Cuervo; se convirtió en la fuente primaria de información dada sus actuales condiciones de desarrollo urbano incompleto o inadecuado y de ilegalidad.

\section{Imagen 1: Localización}
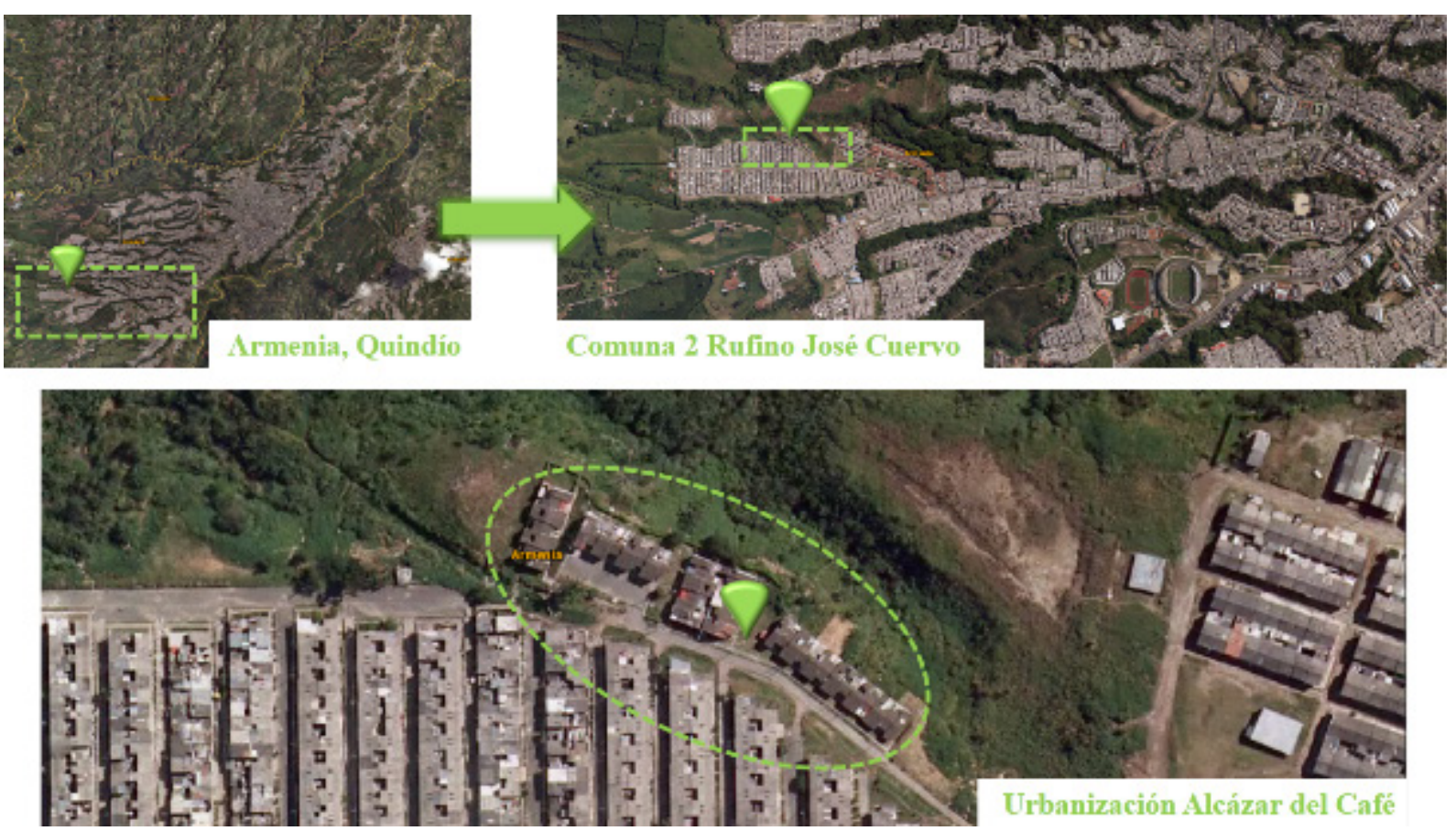

Fuente: SIG Quindio. Montaje. Archivo del Autor. 
Durante el ejercicio, se realizaron recorridos de manera grupal junto con la comunidad que permitieran conocer el espacio físico en el que se trabajó, así como estudiar de manera creativa el espacio que esta comunidad habita. Esta actividad le facilitó al grupo de investigación, adentrarse, vivir, experimentar y observar críticamente en lo que comúnmente sus habitantes no se detienen a pensar porque ya está así establecido. Por su parte, la información secundaria se extrajo de diferentes informes realizados por la Alcaldía Municipal de Armenia en sus secretarías de despacho (Secretaría de Desarrollo Económico, Secretaría de Desarrollo Social), la Oficina Municipal para la Gestión del Riesgo de Armenia- Omger, y del Departamento Administrativo de Planeación Municipal.

\section{Resultados}

Los asentamientos humanos formales con desarrollo incompleto o inadecuado "se caracterizan por ser áreas urbanas constituidas y cuyas condiciones físicas y ambientales no cuentan con el espacio requerido para el desarrollo social de los habitantes" (Vergel, 2010); esta situación se refleja en la falta de espacios de cohesión social que promuevan no solo las relaciones interpersonales sino la cultura, el deporte y la posibilidad de desarrollar actividades económicas secundarias para los ciudadanos. El barrio Alcázar del Café se cataloga como asentamiento humano con desarrollo incompleto, puesto que, aunque no posee orígenes espontáneos, presenta cierto grado de deterioro en su composición físico-espacial, haciéndose evidente en la existencia de infraestructura inadecuada para el tránsito peatonal hacia las zonas perimetrales, la inexistencia de espacios que permitan la divulgación sociocultural tanto de infantes como de adultos, la regulación y relación con el entorno natural circundante, la ausencia de mixtura de usos planificados que permitan el desarrollo endógeno de la comunidad y la ocupación espontanea e informal de terrenos en la zona periférica del sector, produciendo como consecuencia fenómenos sociales adversos para sus pobladores. A continuación se presentan los principales resultados obtenidos, en clave de "Inteligencia Territorial" (Bozzano, Girardot, Cirio, Barrionuevo, \& Gliemmo, 2012), haciendo alusión a la teoría con la que se apuesta por la consolidación de un desarrollo sustentable con la inclusión de los diferentes actores con el fin de validar un conocimiento sobre el territorio a partir de sus identidades, necesidades y sueños.

\section{Identidades: paseo y conversación}

Según la historia del barrio, este fue entregado un año después del terremoto del año 1.999 ocurrido en la región cafetera colombiana, como respuesta habitacional a los damnificados del mismo e hizo parte de un programa del Fondo para la Reconstrucción económica y Social del Eje Cafetero (Forec) llamado "Vitrina Inmobiliaria" que proponía diferentes tipos de vivienda a la cual los damnificados podrían acceder. La Urbanización Alcázar del Café fue un proyecto llevado a cabo por la constructora Conhabitat S.A. quién lo ofertó como parte del denominado grupo 5 del programa mencionado que hacía referencia a un constructor que en ese entonces contara con un lote propio que pudiera ser utilizado para dichos fines. Luego de ser construido, el proyecto fue abandonado sin haber definido y ejecutado a satisfacción los espacios públicos y sin terminar el proceso de legalización y titulación de predios. Este asentamiento humano se compone de 70 unidades habitacionales de hasta $62 \mathrm{~m}^{2}$ con 35 viviendas bi-familiares (Imagen 2) que albergan cerca de 290 habitantes y cuenta con Junta de Acción Comunal desde su fundación.

Con el pasar de los años y tras ver que su barrio no ha sido legalizado, este se convirtió en un lugar donde ya no habitan la mayoría de sus dueños originales, ahora sus habitantes son principalmente arrendatarios. En su interior se percibe un ambiente familiar, sin embargo no existe una unión comunitaria suficiente que permita conocer e interactuar fácilmente con los vecinos; es una población muy guiada al habitar individual de su vivienda. El barrio tiene una vocación de dormitorio. Sus problemáticas sociales y ambientales son principalmente con los barrios colindantes (la fachada etapa I y Jardines de la Fachada) debido a que sus pobladores depositan basuras en los antejardines, presentándose también

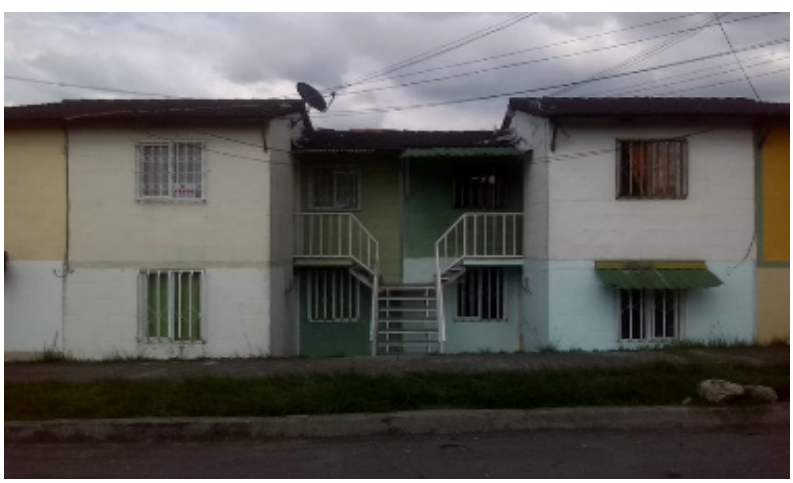

Imagen 2: Vivienda Bi-familiar Tipo. Fuente: Archivo de los autores 
hechos de hurtos en las viviendas lo que ha ocasionado daños en los inmuebles e inseguridad en los habitantes.

\section{Necesidades: diagnosticar para interpretar}

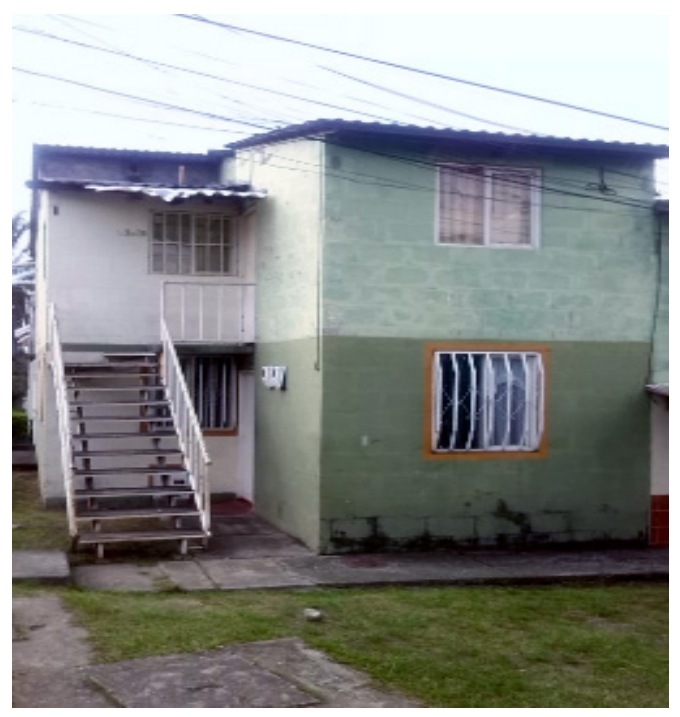

Imagen 3: Vivienda Tipo, Fuente: Archivo de los autores

\section{Físico-espacial}

Desde el punto de vista de la accesibilidad y conectividad, se reconoció que la urbanización cuenta con una vía pavimentada que comunica directamente al barrio La Fachada, por esta no acceden buses, sirve para el acceso peatonal y de vehículos pequeños. Por su parte, se identificó que el barrio no cuenta con equipamientos recreativos, educativos, de salud, comerciales o religiosos; lo que demanda a su población a desplazarse hacia otros sectores para acceder a estos. De igual forma se encontró al interior y de manera colindante con el barrio la existencia de varios lugares sin construir que podrían ser aprovechados para la configuración de espacios al servicio de la comunidad.

Se identificó que todas las viviendas conservan su homogeneidad en la altura edificada, presentan buenas condiciones constructivas dada la materialidad originalmente utilizada (Imagen 3), esto a pesar de las múltiples modificaciones que se han realizado al interior de ellas, las cuales fueron hechas sin el aval normativo requerido dada la falta de titulación de los predios, sin embargo esto no ha frenado las intenciones de mejoramiento y ampliación que se han ejecutado en un alto porcentaje de las viviendas. Este barrio cuenta con una buena cobertura de servicios públicos.

Por su parte, se encontraron solo 2 viviendas donde el uso residencial principal se combina con el comercial siendo destinadas para la venta de víveres. La mayoría de las viviendas (cerca del $85 \%$ ) se encuentran actualmente en lo que se definió como el tercer tipo de ampliación progresiva según las posibilidades establecidas en el diseño inicial; la cual consiste en la posibilidad de contar con un baño, cocina, sala-comedor, dos patios y tres habitaciones, todo ello en $62 \mathrm{~m}^{2}$. Teniendo esto en referencia y una vez se identificó que el promedio de habitantes por vivienda (Gráfica 1) es de 3 a 4 personas se estableció que el nivel de hacinamiento en ellas es normal.

Gráfica 1. Habitantes por vivienda

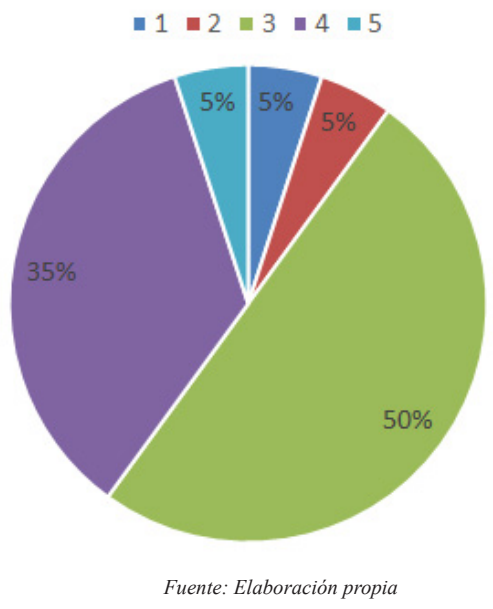

\section{Social y económico}

Además de lo mencionado, se encontró que con relación a los intervalos de edades presentes en el asentamiento humano se habla de que $74 \%$ de la población son personas adultas mientras que los niños y adolescentes representan una minoría para el barrio. Se identificaron a su vez algunos problemas de convivencia entre los vecinos, como lo son la falta de comunicación, integración y de sentido de pertenencia, evidenciando la gran necesidad que tienen de espacios comunitarios, situación que empieza a afectar el comportamiento de los niños.

Se identificó un déficit de espacios públicos, así como una cultura de no ejercicio tanto en personas jóvenes 
como adultas y las pocas personas que se ejercitan deben desplazarse hacia otras partes, debido a que el asentamiento humano no cuenta con un área apta para la recreación, solo cuenta con unos espacios abiertos en mal estado que no tienen un uso definido. En cuanto a los niveles de escolaridad, el $64 \%$ de los habitantes dijeron estar en proceso de formación, la mayoría de adultos obtuvieron su educación hasta la secundaria, por su parte, entre los adolescentes se identificó que estos se encuentran realizando ya sea formación básica secundaria o universitaria; el $72 \%$ de los habitantes que estudian deben desplazarse a otras zonas de la ciudad para adquirir ese servicio, en la mayoría de los casos los estudiantes deben desplazarse a otras comunas y en algunos casos deben desplazarse al otro extremo de la ciudad.

Gráfica 2. Medio de trasporte más utilizado

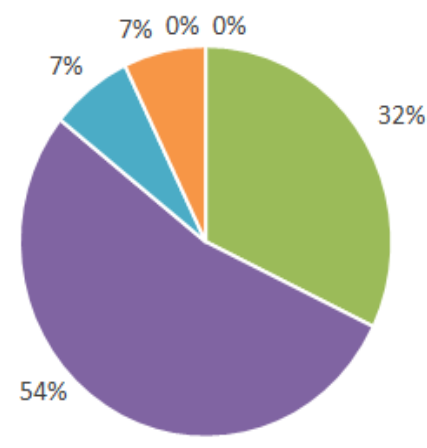

\footnotetext{
- A pie " Bicicleta " Moto " Bus = Taxi " Vehiculo Particular Fuente: Elaboración propia
}

La mayoría de sus habitantes solo habitan las viviendas en las noches ya que tienen sus empleos en el centro de la ciudad. Algunos reciben remesas. El $73 \%$ de los habitantes solo se encuentran presentes en las horas en que descansan. Las amas de casa permanecen en sus viviendas en las mañanas y en las tardes se dedican a la realización de otras actividades; no cuentan con los espacios adecuados para compartir entre vecinos.

En cuanto al trasporte y la movilidad los habitantes de este asentamiento humano manifestaron que suelen desplazarse en bus (Gráfica 2), sin embargo no cuentan con un buen servicio de trasporte público urbano, para abordar una de las dos rutas con las que cuenta el sector hay que desplazarse hasta el barrio vecino.

\section{Ambiental}

En el barrio se encontraron dos tipos de afectaciones ambientales que inciden en los habitantes durante sus actividades cotidianas: la contaminación del suelo y la contaminación del aire.

La contaminación del suelo reconocida con la presencia de residuos sólidos y escombros en los alrededores del barrio siendo una de las principales problemáticas ambientales evidenciadas, esta situación es ocasionada por algunos de sus habitantes y en especial por personas exteriores que utilizan el lugar como basurero. Esto conduce a la contaminación del aire debido a la liberación de compuestos en la materia orgánica que atraen gran variedad de plagas, sumado a la falta de canalización de la quebrada ubicada en la parte posterior del barrio la cual produce malos olores y mosquitos. El asentamiento al estar ubicado en una de las periferias de la ciudad tiene la oportunidad de colindar con un gran espacio natural que es mal aprovechado (Imagen 4).

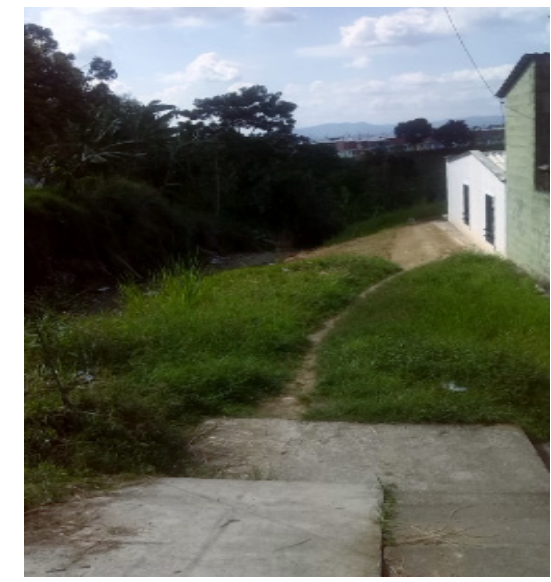

Imagen 4: Sector posterior del barrio, Fuente: Archivo de los autores

\section{Normativo}

Se logró establecer que según el Plan de Ordenamiento Territorial (POT) del Municipio de Armenia (Concejo Municipal de Armenia, 2009) el barrio Alcázar del Café se encuentra regido por la Ficha Normativa 2A, la cual señala que se debe destinar para las áreas comunales en las agrupaciones de vivienda como esta un porcentaje tanto para zonas recreativas de espacio abierto como para zonas de uso comunal bajo cubierta. Esto permite que dentro del barrio se puedan hacer 
propuestas en tal sentido, aprovechando que se cuenta con lugares en su interior y con instrumentos normativos referidos a legalización de asentamientos humanos y al mejoramiento integral del Barrios como lo son: Decreto Nacional 564/2006 (Ministerio de Ambiente, Vivienda y Desarrollo Territorial; Presidencia de la República, 2006) que plantea los lineamientos generales sobre el particular, el Decreto Municipal de Armenia 104/2013 dónde se convoca a los asentamientos humanos en estado de informalidad urbana a presentar documentación; Ley 388/97 (Congreso de la República de Colombia, 1997) sobre ordenamiento territorial en la que se establecen diferentes mecanismos de actuación; el Plan de Desarrollo Municipal de Armenia 2012-2015 (Concejo Municipal de Armenia, 2012) donde se estipula la necesidad de legalizar asentamientos informales en la ciudad; la Política Nacional de Mejoramiento Integral de Barrios -Conpes 3604- (Ministerio de Ambiente, Vivienda y Desarrollo Territorial; Departamento Nacional de Planeación, 2009) en donde se establecen las herramientas generales para la gestión de programas nacionales de mejoramiento de barrios.

\section{Escuchar para crear}

Posteriormente se realizaron ejercicios de cartografía social (Andrade y Santamaría, 1997) y diagnóstico participativo (Gonzáles, 1995) teniendo como referencia al análisis multivariado antes presentado. Estas se ejecutaron con los habitantes del barrio divididos en grupos de edad.

Con los niños se realizaron tres actividades tomadas como referencia de la ONG Architecture sans frontieres del Reino Unido (2012) con las que se buscó la interpretación de ellos frente a necesidades principales, aspectos positivos y negativos que ellos tienen con respecto al lugar que habitan, cómo es y cómo perciben su barrio por medio de actividades lúdicas (Imagen 5)

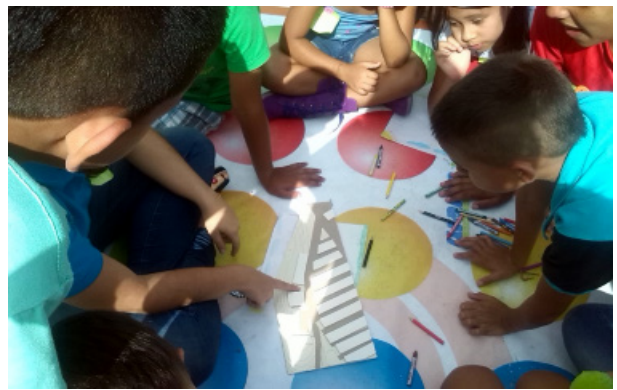

Imagen 5: Actividad con Niños Fuente: Archivo de los autores
- Dibujando mi barrio, por medio de actividades de expresión gráfica.

- Conociendo mi barrio, a partir de preguntas acerca del barrio y de su vivencia en él.

- La vida en mi barrio, por medio de la realización de títeres, que los niños construyeron con elementos como medias, lana, botones, etc. en los cuales representaran a las personas más reconocidas en el barrio.

Al realizar estas actividades quedó muy clara la percepción que los niños tienen del barrio, para ellos el barrio es su hogar y su lugar para hacer amigos, pero también es un lugar lleno de intolerancia en los cuales no pueden jugar debido a que muchos de los adultos no se lo permiten. Se evidenció que para los niños existe un punto de referencia principal dentro del barrio (Imagen 6) y ese punto es el parque como ellos lo llaman, al hacer esta actividad se pudo dar cuenta de que el barrio está física y socialmente fraccionado y que cada habitante solo conoce la zona donde vive.

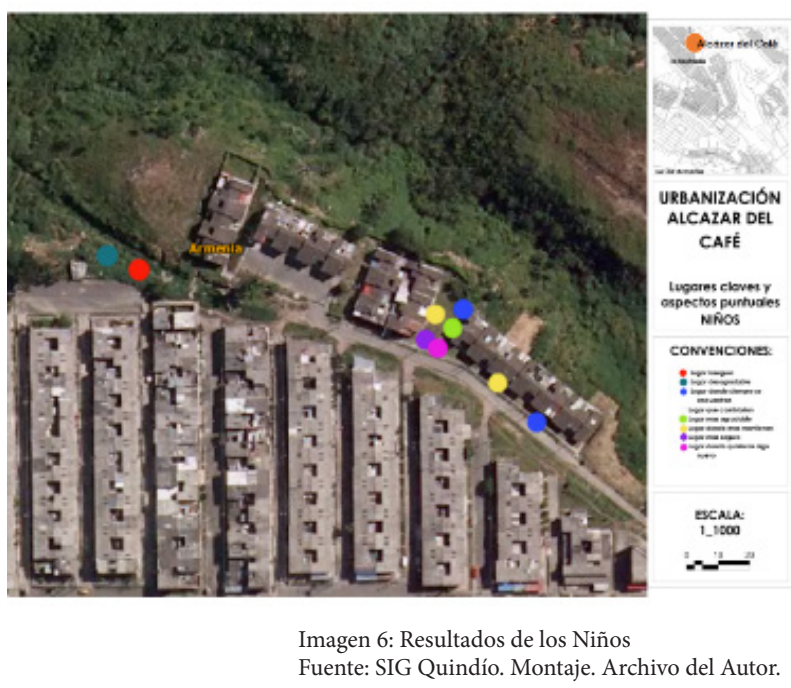

En esta etapa los niños dejaron claro que su mayor necesidad es la de contar con espacios recreativos que les permita mayor libertad y donde sientan que los adultos no los van a regañar o a agredir verbalmente, lugares donde compartir con sus amigos y divertirse dentro de la seguridad que el barrio les ofrece. 
Con los adolescentes se realizaron dos actividades con las que se buscaron sus aportes en el mismo sentido que con el grupo anterior sobre las necesidades, aspectos positivos y negativos que ellos tienen. Se encontró que la mayoría de ellos no conoce en totalidad su barrio; Además, los resultados fueron similares a los arrojados en las actividades con los niños puesto que los jóvenes identifican los mismos sitios que ellos. Por su parte, se encontró que los jóvenes le tienen un nombre a cada espacio particular, están muy apropiados de su urbanización, la sienten suya y ellos más que nadie quisieran que su barrio fuera el mejor de todos y llegaron a proponer algunas ideas para aprovechar los espacios abiertos (Imagen 7).

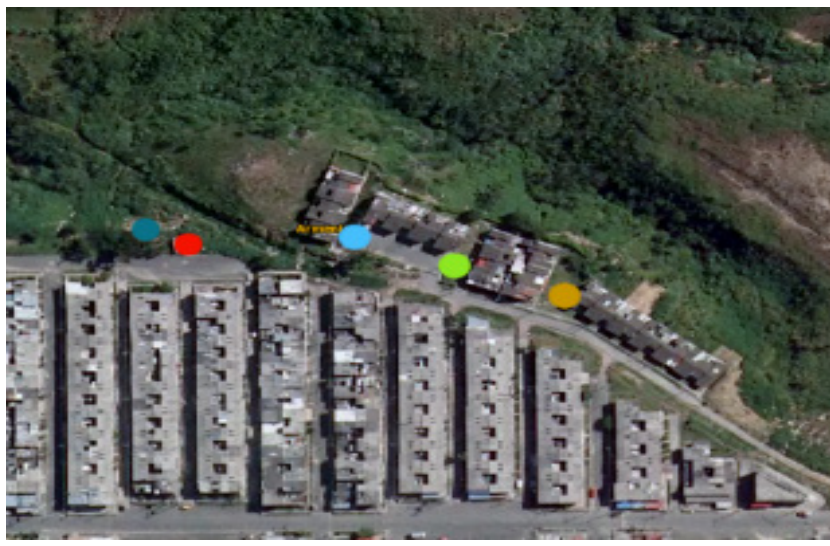

Imagen 7: Resultados de los Adolescentes

Fuente: SIG Quindío. Montaje. Archivo del Autor.

Por su parte, en los diferentes encuentros sostenidos con las personas adultas (Imagen 8 ver pág sig) se pudo comprobar que el sector presenta las falencias urbano-arquitectónicas lógicas de la imposibilidad legal para mejorar los espacios colectivos en el barrio. Esta misma situación se evidencia en el deterioro y la falta de apropiación de las zonas destinadas para el uso común. Por su parte las viviendas han sido modificadas de manera inapropiada por sus propietarios tratando de satisfacer según sus posibilidades las necesidades habitacionales de las familias. Todo ello se manifiesta en algunas circunstancias que reflejan la alteración de la convivencia, la seguridad y las posibilidades de actuar de manera acorde con los parámetros legales.

Además, se evidenció una división del barrio en tres sectores (Imagen 9 ver pág sig), lo que genera un deterioro en los factores de comunicación e integración entre sus habitantes, esto a pesar de contar con lugares donde se podrían dinamizar acciones que superen esta situación y que a su vez pueden ser aprovechados para actividades productivas económicamente para la misma comunidad. De igual manera, se hizo evidente la falta de intervención de los entes gubernamentales en la gestión de la seguridad comunitaria además de la imposibilidad para acceder a ciertos derechos básicos, situación que se hace más evidente por tratarse de un asentamiento ilegal. Se evidenció también la falta de identidad y de apropiación de los habitantes adultos hacia su barrio, observándose una vocación netamente residencial o como lugar dormitorio y debido a que es residido principalmente por inquilinos y por algunos de los propietarios originales de las viviendas, es una situación que dificulta la cohesión necesaria al momento de formulación, implementación y apropiación de alternativas para el mejoramiento urbano arquitectónico del mismo.

\section{Sueños: co-construir para trasformar}

\section{Las propuestas}

Una vez conocidos y analizados los resultados previos y luego de identificar los posibles espacios destinados para el uso colectivo y sus condiciones físicas actuales; se realizaron ejercicios de diseño participativo para definir las estrategias de desarrollo barrial. Se acordó entre el equipo investigador y los participantes de la comunidad que las propuestas urbano-arquitectónicas permitieran la configuración de espacios públicos acordes a las necesidades de la población, sus condiciones socio-económicas y sus verdaderas posibilidades de materialización. Por ello que se plantean intervenciones en diferentes escalas y grados, dando la posibilidad a la comunidad de realizar lo propuesto de manera paulatina y contando con el apoyo permanente del gobierno local.

En tal sentido se establecieron como parámetros de intervención el aprovechamiento de los espacios en desuso tanto al interior como a la periferia del barrio para la apropiación de los vecinos (Ilustración 1). 


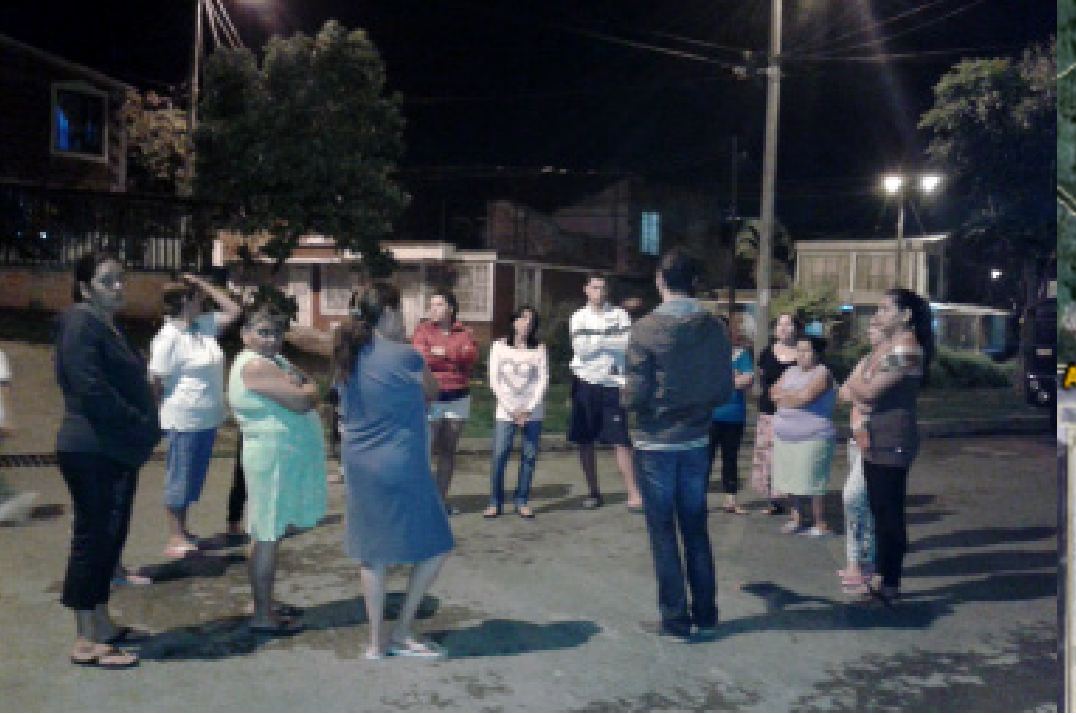

Imagen 8: Conversatorio con adultos, Fuente: Archivo de los autores

Ilustración 1. Premisas de intervención en espacios abiertos

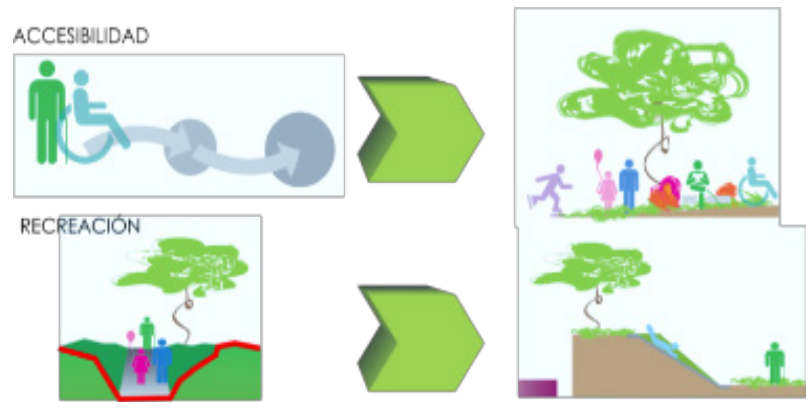

Además se definió la necesidad de adecuar espacios de aprovechamiento colectivo en lugares al aire libre y bajo cubierta (Ilustración 2).

Ilustración 2. Premisas de intervención en espacios cerrados

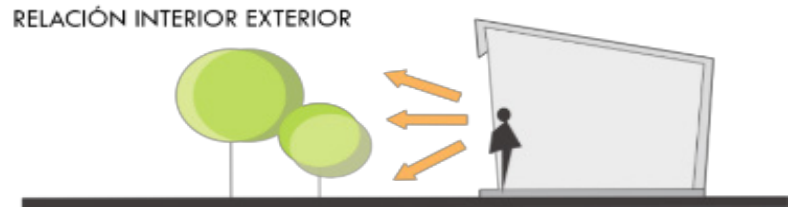

Fuente: Elaboración digital propia de los autores.

Los diseños debían permitir el fácil acceso y disfrute de los habitantes y una permanente relación con el entorno inmediato para facilitar su vigilancia y mantenimiento. Dentro de las propuestas generadas con los habitantes, se plantearon algunas alternativas urbano-arquitectónicas con el fin de suplir las necesidades en 3 ámbitos diferentes (Plano 1).

En el ámbito social se propone la construcción de la caseta comunal, que supla la falta de un lugar de integración para los habitantes, tanto niños como adultos. En segundo lugar, con relación al ámbito recreativo se plantea la proyección de un parque infantil y zonas de recorridos con juegos recreativos, los cuales suplen la necesidad de un lugar para la población infantil donde puedan desarrollarse socialmente, incrementar su madurez intelectual, su fuerza física o su habilidad manual.

Por último en el terreno ambiental se plantea la proyección de huertas urbanas para el uso de la comunidad (Plano 2) siendo estas formuladas como una unidad productiva. En tal sentido, se plantea la formulación de campañas para el cuidado y apropiación del espacio común y público, donde se realicen actividades de limpieza en las zonas contaminadas y los cuidados de estas posteriormente.

Luego del planteamiento inicial se realizó una socialización con la comunidad donde ellos dieron su punto de vista acerca de aspectos importantes en el momento de realizar los diseños definitivos de las propuestas expuestas. Esto evidencia la importancia de involucrar el pensar y el querer de la comunidad hacia la superación de este tipo de falencias que afectan a muchos sectores de la ciudad en pos del mejoramiento de las condiciones de vida, que contengan sustentos teóricos, conceptuales y propositivos, contextualizados no solo numéricamente sino también en el reconocimiento de la comunidad y el derecho que poseen de ser parte activa de 
- Áreas de Cesión Z1

- Áreas de Cesión Z2

- Áreas de Cesión Z3

- Áreas de Cesión Z4

- Propuestas de intervención en áreas de espacio público efectivo

Durante este acompañamiento se ha contado con la receptividad y respaldo permanente de la subdirección de planeación municipal de Armenia, oficina encargada de dar trámite a este tipo de gestiones. En dicha oficina solo resta un documento por parte de la constructora original.

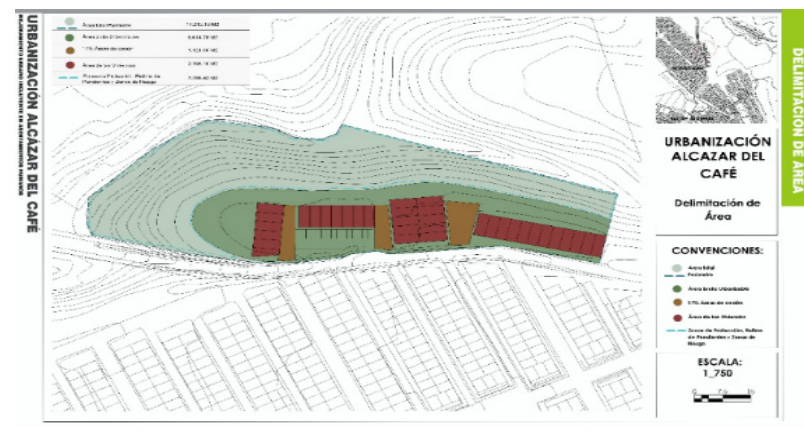

Plano 3: Delimitación de Areas, Fuente: Montaje. Archivo del Autor

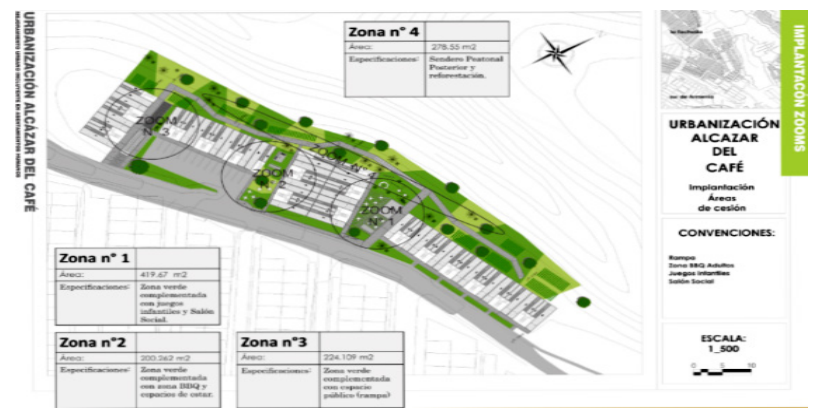

Plano 4: Tratamientos de áreas de cesión, Fuente: Montaje. Archivo del Autor.

\section{Conclusiones}

Los asentamientos humanos formales con desarrollo incompleto o inadecuado como el asentamiento humano Alcázar del Café se caracterizan por ser áreas urbanas constituidas y cuyas condiciones físicas y ambientales no cuentan con el espacio requerido para el desarrollo social de los habitantes; esta situación se refleja en la falta de espacios de cohesión social que promuevan no solo las relaciones interpersonales sino la cultura, el deporte y la posibilidad de desarrollar actividades económicas secundarias para los ciudadanos.

Dadas las condiciones de ilegalidad y de ubicación en la ciudad, se hace evidente la falta de identidad y arraigo de los habitantes hacia su barrio, situación que dificulta la cohesión necesaria en el momento de la formulación, implementación y apropiación de alternativas para el mejoramiento urbano arquitectónico del mismo. A pesar de ello, durante el proyecto de investigación -en los momentos de la formulación y exploración de alternativas y estrategias para el mejoramiento integral del barrio con miras a solucionar problemáticas presentes en su calidad de vida - se evidenció que los niños presentan un nivel de apropiación y de respeto por su ciudad y su entorno diferente que el de los adultos.

Se considera que la propuesta surgida tiene un enfoque desde el cual la arquitectura le brinda sentido y dignidad a los espacios públicos y comunitarios con el fin de que esta se convierta en un dinamizador para el mejoramiento de relaciones, la comunicación y la cohesión entre los vecinos y de ellos con su entorno inmediato.

Por último se puede concluir que los procesos de legalización de barrios se encuentran en nuestro contexto municipal con múltiples dificultades, bien sea porque el tamaño de la problemática dificulta el actuar efectivo de los entes gubernamentales o porque las comunidades a quienes cobija esta situación no cuentan con los recursos técnicos, logísticos y financieros para llevarlos a buen término. El proceso de legalización del asentamiento humano Alcázar del Café, a pesar de las actuales dificultades con el urbanizador inicial, cuenta con avances significativos que a corto plazo permitirá su terminación. Lo anterior gracias a la participación de la academia y a su articulación con una comunidad que requiere y anhela dicho logro.

\section{Referencias bibliográficas}

Andrade M., H., \& Santamaría, G. (1997). Cartografía Social para la planeación participativa. Memorias del Curso: Participación Comunitaria y Medio Ambiente. Proyecto de capacitación para profesiones del Sector Ambiental. Bogotá: Ministerio del Medio Ambiente e ICFES. Recuperado el 10 de 07 de 2007 
Apsan Frediani, A., French, M. A., \& Nuñez Ferrera, I. (2011). Change by design. Building communities through participatory design. New Zealand: Urban Culture Press.

Architecture sans frontières. (2012). Participate. Obtenido de Architecture sans frontières: http:// www.asfparticipate.org/

Bozzano, H., Girardot, J.-J., Cirio, G., Barrionuevo, C., \& Gliemmo, F. (2012). Inteligencia Territorial: Antecedentes en América Latina y Desafios Globales. La Plata: Universidad Nacional de San Juan.

Bru Martín, P., \& Basagoiti R., M. (2003). La Investigación-Acción Participativa como metodología de mediación e integración socio-comunitaria. Revista Comunidad(6). Obtenido de http://www.pacap.net/es/ publicaciones/pdf/comunidad/6/documentos investigacion.pdf

Concejo Municipal de Armenia. (2009). Acuerdo Municipal N. 019 de Noviembre de 2009. "Por medio del cual se adopta el Plan de Ordenamiento Territorial del Municipio de Armenia, para el periodo 2009-2023, Armenia una ciudad de oportunidades". Armenia: Gaceta Municipal de Armenia.

Concejo Municipal de Armenia. (2012). Acuerdo N. $^{o}$ 005 de Mayo de 2012: Plan de Desarrollo "Armenia, un paraíso para invertir, vivir $y$ disfrutar" 2012 - 2015. Armenia: Gaceta Municipal de Armenia.

Congreso de la República de Colombia. (1997). Ley 388 de 1997 sobre ordenamiento territorial. Bogotá D.C.: Imprenta Nacional de Colombia.

CRQ; IGAC; Gobernación del Quindío. (2010). SiG-Quindio. Obtenido de Sistema de infromación geográfica: http://200.21.93.53/ sigquindioii/

El Troudi, H., Harnecker, M., \& Bonilla, L. (2005). Herramientas para la participación. Caracas: MPD.
Gonzáles, E. (1995). Manual sobre participación y organización para la gestión local. Cali: Foro Nacional por Colombia.

Ministerio de Ambiente, Vivienda y Desarrollo Territorial; Departamento Nacional de Planeación. (2009). Documento Conpes 3604: Política de mejoramiento integral de barrios. Bogotá D.C.: Consejo Nacional de Política Economica y Social.

Ministerio de Ambiente, Vivienda y Desarrollo Territorial; Presidencia de la República. (2006). Decreto 564 de 2006. Diario Oficial 46192.

Torres Tovar, C.A. (2009). Ciudad informal colombiana, barrios construidos por la gente. (v. e. Grupo de investigación de procesos urbanos en hábitat, Ed.) Bogotá: Universidad Nacional de Colombia de Bogotá.

Universidad Pontificia Javeriana, Area Metropolitana del Valle de Aburra. (2007). Mejoramiento integral del hábitat para la region metropolitana del Valle de Aburra. Medellin: Artes y letras Ltda.

Vergel T., E. (2010). Asentamientos precarios. Una aproximación para su mejoramiento integral y prevención. DE-ARQ Revista de Arquitectura Universidad del Los Andes.(06), 64 - 81. 
